\title{
ChemComm
}

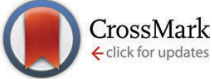

Cite this: Chem. Commun., 2016, 52,697

Received 17th October 2015, Accepted 28th October 2015

DOI: $10.1039 / \mathrm{c} 5 \mathrm{cc} 08617 \mathrm{e}$

www.rsc.org/chemcomm

\section{Bioinspired $\mathrm{pH}$ and magnetic responsive catechol-functionalized chitosan hydrogels with tunable elastic properties $\uparrow$}

\author{
Ali Ghadban, ${ }^{\mathrm{ab}}$ Anansa S. Ahmed, ${ }^{\mathrm{a}}$ Yuan Ping, ${ }^{\mathrm{ab}}$ Ricardo Ramos, ${ }^{\mathrm{ab}}$ Najmul Arfin, ${ }^{\mathrm{ab}}$ \\ Bram Cantaert, ${ }^{a b}$ Raju V. Ramanujan ${ }^{a}$ and Ali Miserez ${ }^{* a b c}$
}

\begin{abstract}
We have developed $\mathrm{pH}$ - and magnetic-responsive hydrogels that are stabilized by both covalent bonding and catechol/Fe ${ }^{3+}$ ligands. The viscoelastic properties of the gels are regulated by the complexation valence and can be used to tune drug release profiles. The stable incorporation of magnetic nanoparticles further expands control over the mechanical response and drug release, in addition to providing magnetic stimuli-responsivity to the gels.
\end{abstract}

The field of hydrogels has rapidly expanded in recent years, ${ }^{1,2}$ with bioinspired and biopolymer-based hydrogels representing an increasingly large fraction of these efforts. A growing theme is the development of hydrogels that are multi-functional, stimuliresponsive, and with tailorable physico-chemical and mechanical properties. $^{3,4}$ Towards obtaining gels with tunable properties, metal coordination bonding has recently emerged as a versatile strategy, notably by exploiting catechol moiety/transition metal complexation., ${ }^{5,6}$ Such ligands are particularly useful to stabilize and modulate gels viscoelastic properties owing to: (i) their $\mathrm{pH}$ dependence that triggers either mono-, bi- or tri-dentate complexes, and (ii) the ability to further tune their properties by simple modification of the transition metal ions used to coordinate the catecholic moieties. ${ }^{7}$ On the other hand, the conformational response of many hydrogels, which would be useful in many applications, remains slow. This limitation can be overcome by preparing magnetic hydrogels, composed of a discrete magnetic phase such as magnetic nanoparticles (MNPs) embedded within a polymeric matrix. ${ }^{8}$ Magnetically-controlled hydrogels provide

\footnotetext{
${ }^{a}$ School of Materials Science and Engineering, Nanyang Technological University, 50 Nanyang Avenue, Singapore 639798, Singapore.

E-mail: ali.miserez@ntu.edu.sg; Fax: +65 6790-9081; Tel: +65 6592-7947

${ }^{b}$ Centre for Biomimetic Sensor Science, Nanyang Technological University,

50 Nanyang Drive, Singapore 637553, Singapore

'School of Biological Sciences, Nanyang Technological University,

60 Nanyang Drive, Singapore 637551, Singapore

$\dagger$ Electronic supplementary information (ESI) available: Materials, methods, analytical techniques, synthesis of hydrogels, ${ }^{1} \mathrm{H}$ NMR of HCA-chit, TEM, DLS, VSM, XRD of MNPs, supplementary rheology data, swelling ratio of hydrogels, videos on hydrogels and drug release study. See DOI: 10.1039/c5cc08617e
}

unique functionalities in biomedical applications such as tissue engineering and drug delivery. For instance, they can be actuated in a controlled manner to prepare $3 \mathrm{D}$ tissue scaffolds during cell proliferation. ${ }^{9,10}$ Alternatively, the behavior of encapsulated cells can also be controlled in vitro by a remotely-activated mechanical stimulus, which is triggered by interactions between the MNPs and an external magnetic field. ${ }^{11}$ Using alternating magnetic fields, MNPs can also be remotely heated to induce: (i) conformational changes to the hydrogels, which can be exploited for the pulsatile release of drugs, ${ }^{12}$ or (ii) hydrophobic-to-hydrophilic phase transitions that can promote large dimensional changes. ${ }^{13}$ Finally, MNPs provide contrast in magnetic resonance imaging studies, enabling therapy and diagnostic (theranostic) related protocols. ${ }^{13,14}$ Thus, the introduction of magnetic responsiveness to the hydrogel increases its versatility to develop a truly multifunctional, multi-stimuli responsive material. ${ }^{7}$

Chitosan, the deacetylated version of the polysaccharide chitin, represents a convenient source of biodegradable biopolymers for a wide range of soft materials, including hydrogels. ${ }^{15}$ It is highly abundant, notably in the waste from the seafood industry; it is soluble in weak acidic solutions; ${ }^{16}$ and it can readily be functionalized to expand its range of functional properties for biomedical applications such as gene delivery and tissue repair. ${ }^{17,18}$ The merging of the two approaches, namely catechol functionalization of chitosan, is quickly emerging as a method of choice to combine the specific advantages of catechol complexation and the ease of processing of chitosan, as exemplified in recent studies. ${ }^{19-22}$ Herein, we explore the potential of catecholfunctionalized chitosan to engineer $\mathrm{pH}$-sensitive and magneticresponsive hydrogels by taking advantage of metal-catechol coordination. Hydrocaffeic acid (HCA) bearing the catechol moiety was used to functionalize chitosan, allowing the modulation of the viscoelastic response of HCA-chitosan gels via $\mathrm{pH}$ triggering and variation of the network mesh size $\xi$, which in turn allowed the tuning of drug release profiles. In order to further expand the multi-functionality of these gels, we then incorporated iron oxide $\left(\gamma-\mathrm{Fe}_{2} \mathrm{O}_{3}\right)$ MNPs in their formulation and established that this strategy adds an additional level of multi-functionality. 


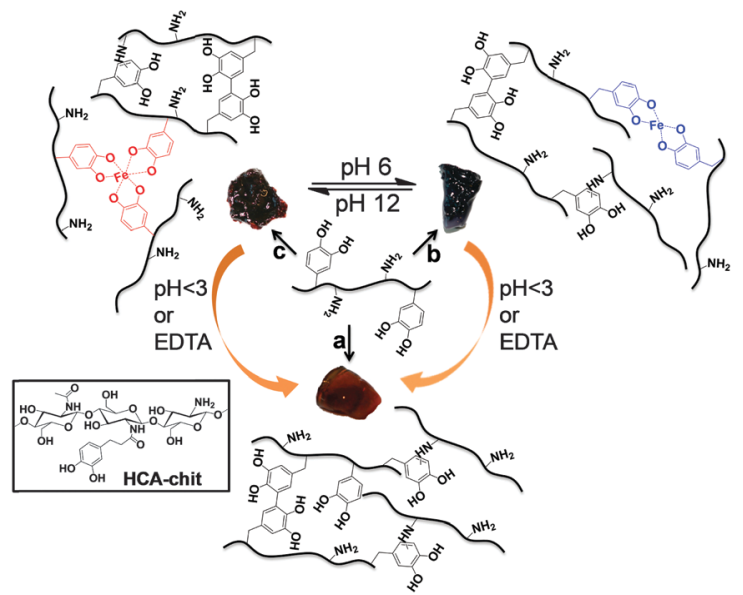

Scheme 1 Schematic of $\mathrm{pH}$-tunable HCA-chitosan hydrogels stabilized by covalent bonding ("chemical gels") and catechol/ $\mathrm{Fe}^{3+}$ complexation ("physical gels"). Gelation conditions used: (a) $\mathrm{NaOH} \mathrm{pH} \mathrm{13;} \mathrm{(b)} \mathrm{FeCl}_{3}$ $\left(\mathrm{Fe}^{3+}: \mathrm{HCA}=1: 3\right), \mathrm{pH} 6$; (c) $\mathrm{FeCl}_{3}\left(\mathrm{Fe}^{3+}: \mathrm{HCA}=1: 3\right), \mathrm{pH}$ 12. Note the yellow-orange color of the covalently cross-linked hydrogel, and the bluepurple and red colors of the metal coordinated cross-linked hydrogels at $\mathrm{pH} 6$ and 12, respectively.

First, it allows further modulation of the mechanical response; second it provides an additional control over drug release kinetics; and third, it confers magnetic responsivity on the gels, opening the door to their usage as stimuli-responsive, biocompatible hydrogels with tunability of drug release kinetics.

First, we functionalized high molecular weight chitosan $\left(M_{\mathrm{w}} \cong 94 \mathrm{KDa}\right)$ with the HCA catecholic moiety, with a degree of substitution of $\sim 15 \%$ as measured by ${ }^{1} \mathrm{H}$ NMR (Fig. S1, ESI $\dagger$ ). The preparation of hydrogels in the absence of MNPs was then investigated. Upon addition of $\mathrm{Fe}^{3+}\left(\mathrm{HCA}: \mathrm{Fe}^{3+}=3: 1\right.$ ), increasing the $\mathrm{pH}$ of the HCA-chitosan solution to 6 and 12 led to the formation of bis- and tris-complexes, respectively. As a control, we also prepared covalently cross-linked hydrogels (hereafter referred to as "chemical hydrogels") by increasing the pH of HCA-chitosan to 13 (Scheme 1). Fig. 1 shows the UV-Vis spectra of the chemical and physical (stabilized by coordination complexation) hydrogels prepared with and without $\mathrm{Fe}^{3+}\left(\mathrm{HCA}: \mathrm{Fe}^{3+}=3: 1\right)$ at different pHs. The presence of an absorbance peak at $280 \mathrm{~nm}$ for HCA-chitosan confirms the successful grafting of HCA to the chitosan backbone. Increasing the $\mathrm{pH}$ from acidic to basic, the color of HCA-chitosan solution changed from transparent to a yellowish/orange color with a simultaneous gel formation, an observation already reported in earlier studies. ${ }^{18}$ Additional peaks at $\sim 260$ and $\sim 350 \mathrm{~nm}$ appeared, which can be attributed to covalent crosslinking resulting from either catechol-catechol or amino-quinone bond formation, respectively. ${ }^{18,22}$ Conducting the gelation in the presence of $\mathrm{Fe}^{3+}$ at $\mathrm{pH} 6$ and 12, blue/purple and red-colored hydrogels were observed, with absorbance peaks at 550 and $495 \mathrm{~nm}$ associated with the formation of bis- and triscomplexes, respectively. The latter result is in agreement with recent reports on catechol-functionalized PEG and chitosan., ${ }^{5,22}$ The presence of chemical crosslinks is inevitable under such conditions due to the high molecular weight of chitosan

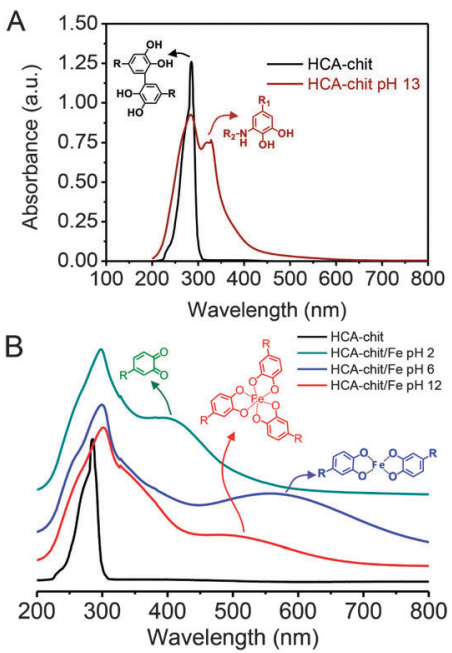

Fig. 1 UV-Vis monitoring of hydrogel formation for HCA-chitosan at different $\mathrm{pH}$ in the absence $(\mathrm{A})$ and presence $(\mathrm{B})$ of $\mathrm{Fe}^{3+}\left(\mathrm{HCA}: \mathrm{Fe}^{3+}=3: 1\right)$.

(resulting in abundant free amino groups), the high degree of substitution (DS $\sim 15 \%$ ), and the redox effect of $\mathrm{Fe}^{3+}$. This is also reflected by the appearance of peaks at $\sim 260$ and $350 \mathrm{~nm}$. It is worth mentioning that the mono-complex associated with a green color formation was observed when the $\mathrm{pH}$ of the HCA-chitosan solution was increased from $\sim 2$ to 3-4 in the presence of $\mathrm{Fe}^{3+}$ (data not shown). The visco-elastic properties of the synthesized hydrogels were characterized two hours after gelation by rheology in the dynamic mode at $20{ }^{\circ} \mathrm{C}$ (Fig. 2). In the tested frequency range, HCA-chitosan (without $\mathrm{Fe}^{3+}$ ) at pH 13 showed the characteristics of a soft hydrogel, with a constant storage modulus $G^{\prime}$ of $c a .500 \mathrm{~Pa}$. The damping factor $\left(\tan \delta=G^{\prime \prime} / G^{\prime}\right)$ was approximately 1 below $0.1 \mathrm{~Hz}$, implying network properties at the boundary between fluid- and solidlike gel. However, above $0.1 \mathrm{~Hz}$ the gel was solid-like, with $G^{\prime} / G^{\prime \prime}>1$. The addition of $\mathrm{Fe}^{3+}$ greatly increased the storage modulus of the gels, which increased from 6 to $c a .20 \mathrm{KPa}$ (at $1 \mathrm{~Hz}$ ) as the $\mathrm{pH}$ of the $\mathrm{HCA}-$ chitosan solution was increased from 6 to 12. At $\mathrm{pH} 3$ in the presence of $\mathrm{Fe}^{3+}, G^{\prime}$ values were considerably lower ( $<1 \mathrm{~Pa}$, Fig. S3, ESI $\dagger$ ). This increase in stiffness can unambiguously be attributed to catechol $/ \mathrm{Fe}^{3+}$ complexation since no other bonds are introduced into the network. As previously reported, ${ }^{23,24}$ increasing the $\mathrm{pH}$ modifies the nature of the coordination complexes between the catechol side-chains and $\mathrm{Fe}^{3+}$, with mono-complexes present below $\mathrm{pH} 3$, bis-complexes at $\mathrm{pH} 6$ and tris-complexes being the dominant species at $\mathrm{pH}$ 12. Using the classical relationship between storage modulus and mesh size $\left(G^{\prime} \sim k_{\mathrm{B}} T / \xi^{3}\right)$, we determined that $\xi$ decreased from $c a .22 \mathrm{~nm}$ at $\mathrm{pH} 13$ in the absence of $\mathrm{Fe}^{3+}$ down to $10 \mathrm{~nm}\left(\mathrm{pH} 6, \mathrm{Fe}^{3+}\right)$ and $5 \mathrm{~nm}\left(\mathrm{pH} \mathrm{12,} \mathrm{Fe}^{3+}\right)$, suggesting a direct correlation between the complexation state and the mesh size (Fig. 2B).

To assess the reversibility of the gelation process, the triscomplex hydrogel was treated with either $\mathrm{HCl}$ or EDTA overnight. The final $\mathrm{pH}$ of $\mathrm{HCl}$ treated hydrogel solution was found to be 5.5. As a control, the chemical hydrogel was also treated 
A

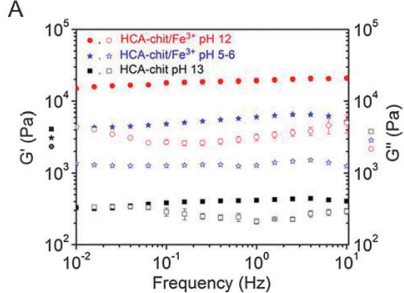

C

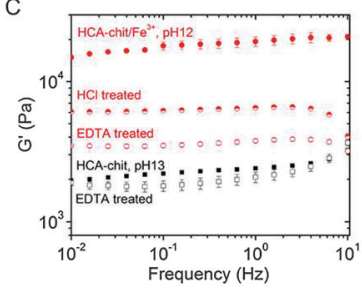

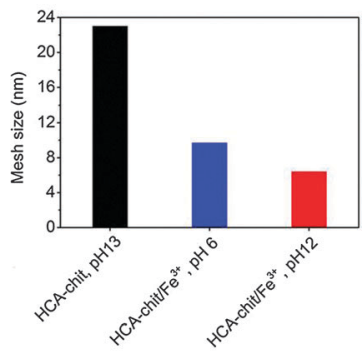

D

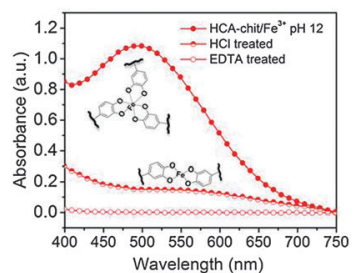

Fig. 2 Rheological properties of synthesized hydrogels (A) in the absence and presence of $\mathrm{Fe}^{3+}$ at various $\mathrm{pHs}$. (B) Estimated mesh size $\xi$ for the different gels. (C) Rheological properties and (D) UV-Vis spectra of physicals gels after treatment with $\mathrm{HCl}$ or EDTA.

with EDTA. The EDTA-treated chemical hydrogels exhibited a storage modulus comparable to that of the initial hydrogel, as expected, given the absence of metallic cations in the initial gel (Fig. 2C). On the other hand, the tris-complex treated gel featured a drop in stiffness, with $G^{\prime}$ decreasing from $\sim 20 \mathrm{KPa}$ to $\sim 6.5$ and $\sim 3.7 \mathrm{KPa}$ (at $1 \mathrm{~Hz}$ ) after $\mathrm{HCl}$ and EDTA treatments, respectively. The UV-Vis analysis supports this finding: a peak at $570 \mathrm{~nm}$ appeared after $\mathrm{HCl}$ treatment corresponding to the bis-complex at this lower $\mathrm{pH}$. Treating the gel with EDTA showed no evidence for any absorbance peak above $450 \mathrm{~nm}$, confirming the removal of all $\mathrm{Fe}^{3+}$ from the gel (Fig. 2D). The higher storage modulus of this hydrogel compared to that of the chemical hydrogel may be attributed to the formation of chemical crosslinks concomitantly taking place during metal chelation, leading to a gel with a higher covalent cross-link density. Collectively, these results indicate that the viscoelastic response of HCA-chitosan gels can be reversibly modulated by chelation or re-incorporation of metal ions.

We previously reported HCA-chitosan films reinforced with MNPs, in which the mechanical properties of the films were enhanced through load transfer from the functionalized-chitosan to MNPs via coordination interactions between catechol and $\mathrm{Fe}_{2} \mathrm{O}_{3}{ }^{20}$ To confer dual $\mathrm{pH}$ and magnetic responsiveness, MNP particles were added to the hydrogels. To this end, HCA-chit were mixed with $\mathrm{Fe}_{2} \mathrm{O}_{3}$ MNPs (2.5 and $5 \mathrm{wt} \%$ ) and gelled by increasing the $\mathrm{pH}$ in the absence or presence of $\mathrm{Fe}^{3+}\left(\mathrm{HCA}: \mathrm{Fe}^{3+}=3: 1\right)$, to yield both chemical and physical hybrid hydrogels. The chemical hydrogels featured a light-brown visual appearance consistent with the color of maghemite $\mathrm{Fe}_{2} \mathrm{O}_{3}$, whereas the physically cross-linked hydrogels maintained a dark red color, suggesting a faster kinetics of complexation for $\mathrm{Fe}^{3+} /$ catechol than for $\mathrm{Fe}_{2} \mathrm{O}_{3} /$ catechol. The addition of 2.5 and $5 \mathrm{wt} \%$ of MNPs to the physical gels resulted in a linear increase in the storage modulus with MNP content of up to $26 \mathrm{kPa}$ at $1 \mathrm{~Hz}$ (Fig. 3A and Fig. S4A, ESI $\dagger$ ). However, we note that the average mesh size $\xi$ estimated from $G^{\prime} \sim k_{\mathrm{B}} T / \xi^{3}$ decreased only
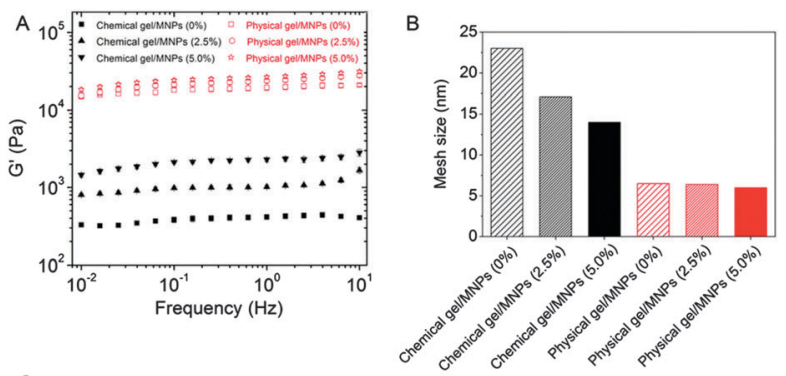

C
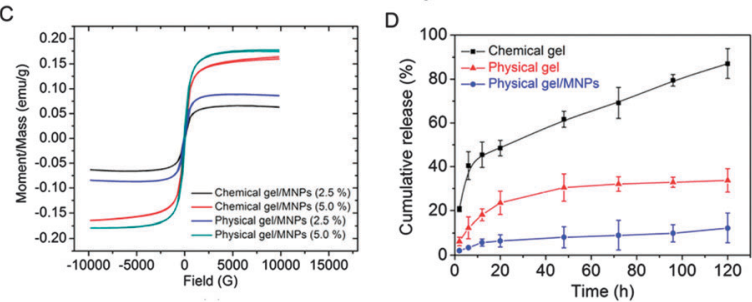

Fig. 3 Rheological, magnetic, and drug-release properties of MNPsloaded hydrogels. (A) Frequency sweep experiments of magnetic chemical and physical hydrogels. (B) Estimated mesh size $\xi$ for the different gels. (C) VSM of chemical and physical magnetic hydrogels. (D) Time-dependent release of dextran-FITC $\left(M_{\mathrm{w}}: 20000\right)$ from different hydrogel at $\mathrm{pH} 12$. The data represent the mean $\pm \mathrm{SD}(n=3)$.

moderately upon addition of MNPs (Fig. 3B), suggesting that catechols favour interactions with $\mathrm{Fe}^{3+}$ and that MNPs primarily act as fillers in the physical gels but do not specifically interact with MNPs. In contrast, the relative increase of $G^{\prime}$ with MNP content for chemical gels was more distinct - though lower in absolute values than for the physical gels - and is reflected by the more pronounced relative decrease in mesh size upon addition of MNPs (Fig. 3B). In the absence of $\mathrm{Fe}^{3+}$, HCA moieties in the chemical gels are free to coordinate the surface of $\mathrm{Fe}_{2} \mathrm{O}_{3}$, thus allowing load transfer from the soft chitosan-HCA matrix to the stiff MNPs. ${ }^{20}$ The increase in $G^{\prime}$ may also be assessed in terms of decreased swelling upon addition of MNPs. The storage modulus of swollen networks is well-established to exhibit an inverse power law dependence on the swelling ratio $Q\left(G^{\prime} \sim Q^{-m}\right),{ }^{25}$ where $m$ is in the range of 2.2 to 3 depending on the solvent. To verify this dependency, we plotted $G^{\prime}$ vs. $Q$ (Fig. S4B, ESI $\dagger$ ) and obtained $G^{\prime} \sim Q^{-2.9}$ for chemical/ MNPs gels, which is in good agreement with the power law predicted for theta solvents and similar to that measured for various PEG hybrid hydrogels. ${ }^{26,27}$ In contrast, in physical/MNPs hydrogels a weaker dependence of $G^{\prime}$ on swelling was observed with $G \sim Q^{-1.2}$, which is consistent with a network where the catecholic moieties are saturated with $\mathrm{Fe}^{3+}$ coordination bonds and thus less available for water uptake. In summary, these data reveal a distinct dependency of $G^{\prime}$ on the MNP content, which can be attributed to substantial differences in the swelling behaviour in the presence or absence of catechol $/ \mathrm{Fe}^{3+}$ coordination bonds.

Vibrating Sample Magnetometer (VSM) measurements were conducted in order to confirm the magnetism of both chemical and physical hydrogels. Fig. 3C illustrates the magnetization versus magnetic field ( $\mathrm{M}-\mathrm{H}$ loop) for the chemical and physical hydrogels at various weight fractions of MNPs. All hydrogels displayed a low hysteresis behavior similar to native $\mathrm{Fe}_{2} \mathrm{O}_{3}$ (Fig. S2D, ESI $\dagger$ ). The magnetic property values of the hydrogels are fully consistent with 
the weight fraction of MNPs in the gels. No change was observed in the magnetic properties even after maintaining the gels in water for 10 days (data not shown). This indicates that the MNPs remained entrapped within the gel without further oxidation. For the same weight fraction of MNPs, physical gels exhibited slightly higher values $\left(\sim 0.18 v s .0 .16\right.$ and $0.09 v s .0 .07 \mathrm{emu} \mathrm{g}^{-1}$ at 5 and $2.5 \mathrm{wt} \%$, respectively), which can be attributed to the smaller swelling ratio of physical gels compared to their chemical analogues (Fig. S5, ESI $\dagger$ ). A video illustrating the magnetically-induced shape morphing of the magnetic hydrogels is provided in the ESI. $\dagger$

In addition to tuning the mechanical properties, MNP hydrogels can also generate additional stress and/or strain in DC magnetic fields, while an AC field could generate a temperature change, enabling thermal triggering. The quick magnetic responsivity could thus be used as implantable magnetic gels containing drugs or cells, which could be delivered on-demand by external stimuli. ${ }^{28}$ To specifically test whether our gels could entrap and release drugs in a controlled manner, both low- and high-molecular weight drugs were incorporated in the gel prior to gelation, and we reasoned that changes in the coordination state would allow the tuning of the release profile. Both physical and chemical gels were able to efficiently entrap rhodamine $\mathrm{B}$, a low-molecular-weight dye that is often used as a probe molecule for drug release studies, after gel formation (Fig. S6, ESI $\dagger$ ). We further used fluorescein isothiocyanate (FITC)-dextran as a macromolecular model drug to evaluate the release kinetics of the drug-loaded gels. As shown in Fig. 3D, whereas the chemical gel released FITC-dextran rapidly, the physical gel exhibited moderate release rate under the same conditions. The difference in release kinetics between both gels is attributed to the mesh size differences (Fig. 2B), which is believed to play an important role in gel diffusivity. ${ }^{29}$ Specifically, physical gels form a denser network reinforced by tris-catechol $/ \mathrm{Fe}^{3+}$ complexes as compared to the chemical gel, which is stabilized by a lower cross-link density of covalent bonds. With the incorporation of MNPs into the physical gels, marginal FITC-dextran release $(<15 \%)$ was observed within $120 \mathrm{~h}$, indicating that the release profile could be further modulated. Since swelling is known to directly influence drug release kinetics, ${ }^{30}$ we attribute the slower release of FITC-dextran in MNP-loaded physical gels to their reduced swelling degree. We also noticed that the release kinetics of the physical gels (with or without MNP incorporation) is $\mathrm{pH}$-dependent. At $\mathrm{pH} 6$, both physical gels exhibited a faster release rate than that at $\mathrm{pH} 12$ (Fig. S7, ESI $\dagger$ ). Collectively, these results indicate that the release of cargos from physical hydrogels can be manipulated by $\mathrm{pH}$ and $\mathrm{Fe}_{2} \mathrm{O}_{3}$ nanoparticle incorporation via variation of mesh size and swelling degree. Expanding this multi-functionality with a modulated mechanical response triggered by $\mathrm{pH}$-dependence metal/ catechol complexation open further opportunities, for instance to adjust the visco-elastic properties of the magnetic hydrogels to specific micro-environments or tissues. Magnetic hydrogels also constitute an interesting approach to design tissue scaffolds for bone and muscle tissue engineering, where the application of stress is required for cell differentiation. ${ }^{31}$ Finally, magnetic fields could be exploited to tailor the mechanical properties of the scaffold to suit optimal growth conditions, while the $\mathrm{pH}$ responsiveness could be used to trigger the release of growth factors at the appropriate times and/or spatial locations. Although the cytotoxicity of these stimuliresponsive gels remains to be assessed, recent studies have indicated a low cytotoxicity of metal coordination reinforced gels using vanadium ions as ligand centers, ${ }^{32}$ suggesting a promising alternative to $\mathrm{Fe}^{3+}$ cross-linked gels should the latter prove cytotoxic.

This research was funded by the Singapore National Research Foundation (NRF) through a NRF Fellowship awarded to A.M.

\section{Notes and references}

1 D. Seliktar, Science, 2012, 336, 1124-1128.

2 C. J. Kearney and D. J. Mooney, Nat. Mater., 2013, 12, 1004-1017.

3 T. L. Sun, T. Kurokawa, S. Kuroda, A. Bin Ihsan, T. Akasaki, K. Sato, A. Haque, T. Nakajima and J. P. Gong, Nat. Mater., 2013, 12, 932-937.

4 X. Zhao, Soft Matter, 2014, 10, 672-687.

5 N. Holten-Andersen, M. J. Harrington, H. Birkedal, B. P. Lee, P. B. Messersmith, K. Y. C. Lee and J. H. Waite, Proc. Natl. Acad. Sci. U. S. A., 2011, 108, 2651-2655.

6 M. S. Menyo, C. J. Hawker and J. H. Waite, Soft Matter, 2013, 9, 10314-10323.

7 N. Holten Andersen, A. Jaishankar, M. Harrington, D. Fullenkamp, G. DiMarco, L. He, G. McKinley, P. Messersmith and K. Y. C. Lee, J. Mater. Chem. B, 2014, 2, 2467-2472.

8 G. Bayramoglu, B. Altintas and M. Y. Arica, Appl. Microbiol. Biotechnol., 2013, 97, 1149-1159.

9 B. S. Kim, J. Nikolovski, J. Bonadio and D. J. Mooney, Nat. Biotechnol., 1999, 17, 979-983.

10 J. R. Mauney, S. Sjostorm, J. Blumberg, R. Horan, J. P. O'Leary, G. Vunjak-Novakovic, V. Volloch and D. L. Kaplan, Calcif. Tissue Int., 2004, 74, 458.

11 H. Fujita, K. Shimizu, Y. Yamamoto, A. Ito, M. Kamihira and E. Nagamori, J. Tissue Eng. Regener. Med., 2010, 4, 437-443.

12 T. Hoare, J. Santamaria, G. F. Goya, S. Irusta, D. Lin, S. Lau, R. Padera, R. Langer and D. S. Kohane, Nano Lett., 2009, 9, 3651-3657.

13 S. Purushotham and R. V. Ramanujan, Acta Mater., 2010, 6, 502-510.

14 S. Purushotham, P. E. J. Chang, H. Rumpel, I. H. C. Kee, R. T. H. Ng, P. K. H. Chow, C. K. Tan and R. V. Ramanujan, Nanotechnology, 2009, 20, 305101.

15 M. Rinaudo, Prog. Polym. Sci., 2006, 31, 603-632.

16 M. Rinaudo, G. Pavlov and J. Desbrieres, Polymer, 1999, 40, 7029-7032.

17 Y. Ping, C. D. Liu, G. P. Tang, J. S. Li, J. Li, W. T. Yang and F. J. Xu, Adv. Funct. Mater., 2010, 20, 3106-3116.

18 J. H. Ryu, Y. Lee, W. H. Kong, T. G. Kim, T. G. Park and H. Lee, Biomacromolecules, 2011, 12, 2653-2659.

19 K. Kim, J. H. Ryu, D. Y. Lee and H. Lee, Biomater. Sci., 2013, 1, 783-790.

20 O. Zvarec, S. Purushotham, A. Masic, R. V. Ramanujan and A. Miserez, Langmuir, 2013, 29, 10899-10906.

21 J. H. Ryu, S. Jo, M.-Y. Koh and H. Lee, Adv. Funct. Mater., 2014, 24, 7709-7716.

22 P. S. Yavvari and A. Srivastava, J. Mater. Chem. B, 2015, 3, 899-910.

23 M. Krogsgaard, M. A. Behrens, J. S. Pedersen and H. Birkedal, Biomacromolecules, 2013, 14, 297-301.

24 B. P. Lee and S. Konst, Adv. Mater., 2014, 26, 3415-3419.

25 K. Urayama, T. Kawamura and S. Kohjiya, J. Chem. Phys., 1996, 105, 4833-4840.

26 M. P. Lutolf and J. A. Hubbell, Biomacromolecules, 2003, 4, 713-722.

27 A. T. Metters, C. N. Bowman and K. S. Anseth, AIChE J., 2006, 47, 1432-1437.

28 X. Zhao, J. Kim, C. A. Cezar, N. Huebsch, K. Lee, K. Bouhadir and D. J. Mooney, Proc. Natl. Acad. Sci. U. S. A., 2010, 108, 67-72.

29 S. Lee, X. Tong and F. Yang, Acta Biomater., 2014, 10, 4167.

30 J. Berger, M. Reist, J. M. Mayer, O. Felt, N. A. Peppas and R. Gurny, Eur. J. Pharm. Biopharm., 2004, 57, 19-34.

31 Y. Li, G. Huang, X. Zhang, B. Li, Y. Chen, T. Lu, T. J. Lu and F. Xu, Adv. Funct. Mater., 2013, 23, 660-672.

32 J. P. Park, I. T. Song, J. Lee, J. H. Ryu, Y. Lee and H. Lee, Chem. Mater., 2015, 27, 105-111. 